\title{
Ein Satz über die zweite Funktionenklasse.
}

\section{Von Celestyn Burstin in Wien.}

Nach Baire ${ }^{1}$ ) heißt eine Funktion von nullter Klasse, wenn sie stetig ist, von erster Klasse, wenn sie Grenze von stetigen Funktionen ist, ohne selbst stetig zu sein, und von zweiter Klasse, wenn sie Grenze von Funktionen erster Klasse ist, ohne selbst eine Funktion nullter oder erster Klasse zu sein. Für die Funktionen nullter Klasse gilt bekanntlich folgender Satz: A us der Gesamtheit aller stetigen Funktionen läbt sich eine abzählbare Menge von stetigen Funktionen $f_{1}(x), f_{2}(x), \ldots f_{n}(x) \ldots$ herausgreifen, welche folgende Eigenschaft besitzt: ist $\varphi(x)$ irgend eine stetige Funktion, so kann man aus der Menge $f_{1}(x), f_{2}(x) \ldots f_{n}(x) \ldots$ eine Teilmenge $f_{n_{1}}(x), f_{n_{2}}(x), \ldots f_{n_{n}}(x), \ldots$ herausgreifen, so dab diese Teilmenge gleichmäßig gegen die Funktion $\varphi(x)$ konvergiert. ${ }^{2}$ )

In dieser Arbeit wollen wir erstens zeigen, daßb für Funktionen erster Klasse ein ähnlicher Satz gilt, zweitens, daß für die Funktionen zweiter Klasse keine abzählbare Menge im Sinne $L$ meßbarer Funktionen existiert, welche die Eigensehaft hätte, daß jede Funktion der zweiten Klasse durch irgend eine Teilfolge dieser Funktionenmenge gleichmä lo ig approximierbar wäre.

Wir führen zunächst einige bekannte Sätze an, auf die wir uns nachher stützen werden.

1. Satz: Ist eine im Sinne $B^{3}$ ) meBbare Funktion $f(x)$ gegeben, welehe a uf einer Menge $M$ rom Inhalt $x>0$ definiertist, so gibtzujeder positiven $\mathrm{Zahl} \alpha$, wobei $0<\alpha<x$ ist, eine perfekte Menge $P_{\alpha}$ vom Inhalt $x-\alpha$, a uf welcher $f(x)$ stetig ist.

Um den Satz zu beweisen, führen wir einen Hilfssatz an:

Hilfssatz: Konvergiert eine Funktionenfolge $\varphi_{1}(x)$, $\varphi_{2}(x) \ldots \varphi_{n}(x) \ldots$ gegen eine Funktion $f(x)$ auf der Menge $M$ vom Inhalt $x>0$, so gibtzu jeder positiven

1) R. Baire: Acta mathematica, Bd. 30, Seite 1.

2) Den Satz hat zuerst bewiesen Weierstraß.

s) Unter "im. Sinne $B$ meßbare Funktionen" versteht man die Funktionen der Menge $E$. "Die Menge $E$ hat folgende Eigenschaften 1 . sie enthält alle stetigen Funktionen, 2. sie enthält mit den Funktionen $\varphi_{1}(x), \varphi_{2}(r) \ldots \varphi_{n}(x) \ldots$ auch ihren limes superior und inferior, 3. sie ist die kleinste Menge, welche die Eigenschaften 1,2 besitzt. 
Zahl $\alpha$, wobei $0<\alpha<x$ ist, eine perfekte Menge $P_{a}$, welche in der Menge $M$ enthalten ist und vom Inhalt $x-\alpha$ ist, wo die Funktionenfolge $\varphi_{1}(x), \varphi_{2}(x) \ldots \varphi_{n}(x) \ldots$ gleichmäBig konvergiert.

In der Tat, sei $\varepsilon_{1} \geqq \varepsilon_{2} \geqq \ldots \geqq \varepsilon_{n} \geqq \ldots$ eine Folge positiver Zahlen, wo $\lim _{n=\infty} \varepsilon_{n}=0$ ist, dann konstruieren wir zu einer zweiten Folge positiver Zahlen $\alpha_{1} \alpha_{2} \ldots \alpha_{n} \ldots$, wo $\sum_{n=1}^{\infty} \alpha_{n}<\alpha$ ist, eine neue Folge positiver Zahlen $\alpha_{1}^{\prime}, \alpha_{2}^{\prime} \ldots \alpha_{n}^{\prime} \ldots$, für welche erstens $\alpha_{n}^{\prime}>\alpha_{n}$ ist und zweitens

ist,

$$
\sum_{n=1}^{\infty} \alpha_{13}^{\prime}=\alpha
$$

Da die Funktionenfolge $\varphi_{1}(x), \varphi_{2}(x), \ldots \varphi^{n}(x), \ldots$ auf der Menge $M$ konvergiert, so gibt es bekanntlich zu jeder positiven Zahl $\varepsilon$ und $\alpha$ eine naturliche Zahl $n$ und eine Menge $M_{\alpha}$ vom Inhalt $x-\alpha$, so dab:

$$
\left|\varphi_{n}(x)-f(x)\right|<\varepsilon
$$

ist wenn $m \geqq n$ ist, und wenn $x$ ein Punkt der Menge $M_{\alpha}$ ist, wobei $M_{o}$ in der $\overline{\bar{M}}$ enge $M$ enthalten ist.

Es folgt daraus, dab zu jeder positiven Zahl $\varepsilon_{1}$ und $\alpha_{1}$ eine natürliche Zahl $n_{1}$ und eine Menge $M_{1}$ existiert derart, daß

$$
(I)\left|\varphi_{m}(x)-f(x)\right|<\varepsilon_{1}
$$

ist, wenn $m \geq n_{1}$ ist und wenn $\dot{x}$ ein Punkt der Menge $M_{1}$ ist, wo die Menge $M_{1}$ in der Menge $M$ enthalten ist und vom Inhalt $x-\alpha_{1}$ ist. Innerhalb der Menge $M_{1}$ gibt es eine perfekte Menge $P_{1}$ vom Inhalt $\left.x-\alpha_{1}^{\prime}<x-\alpha_{1} .{ }^{1}\right)$ Es gibt also die Ungleichung $(I)$ für die Menge $P_{1}$.

Allgemein gibt es zu den positiven Zahlen $\varepsilon_{x}$ und $\alpha_{i}$ eine natürliche Zahl $n_{*}$ und eine Menge $M_{*}$ derart, daß

$$
(I I)\left|\varphi_{m}(x)-f(x)\right|<\varepsilon_{\%}
$$

ist, wenn $m \geqq n_{x}$ ist, und wenn $x$ ein Punkt der Menge $M_{x}$ ist, wobei die Menge $I M_{x}$ in der perfekten Menge $P_{x-1}$ enthalten ist, und wo die Menge $M_{z}$ vom Inhalt $x-\alpha_{1}^{\prime}-\alpha_{2}^{\prime}-\ldots-\alpha_{z-1}^{\prime}-\alpha_{x}$ ist. Innerhalb der Menge $M_{z}$ gibt es eine perfekte Menge $P_{x}$ vom Inhalt $x-\alpha_{1}^{\prime}-\alpha_{2}^{\prime}-\ldots-\alpha_{x-1}^{\prime}-\alpha_{x}^{\prime}<x-\alpha_{1}^{\prime}-\alpha_{2}^{\prime}-\ldots \alpha_{x-1}^{\prime}-\alpha_{x}$. Es gilt also die Ungleichung $(I I)$ desto eher für die perfekte Menge $P_{n}$

Bezeichnen wir den Durchschnitt aller perfekten Mengen $P_{1}$, $P_{2}, P_{3}, \ldots P_{n t} \ldots$ mit $Q_{\alpha}$, so ist erstens die Menge $Q_{\alpha}$ abgeschlossen

1) C. Burstin: Eigenschaften meßbarer und nichtmeßbarer Mengen. Sitzungsberichte der Kajs, Akad. d. Wiss. in Wien, math.-naturw. Klasse. Bd. 123, Abt. 2 a, Seite 1526 . 
und zweitens vom Inhalt $\lim _{n=\infty}\left(x-\sum_{m=1}^{m=n} \alpha_{m}^{\prime}\right)=x-\alpha$. Die Menge $Q_{a}$ ist eine Summe einer abzählbaren Menge $A$ und einer perfekten Menge $P_{\alpha}$. Es ist also die Menge $P_{\alpha}$ auch rom Inhalt $x-\alpha$. Auf der perfekten Menge $P_{\alpha}$ ist die Funktionenfolge $\varphi_{1}(x), \varphi_{2}(x), \ldots$, $\varphi_{n}(x), \ldots$, wie man ohne weiteres einsehen kann, gleichmälig konvergent.

Dadurch ist der Hilfssatz bewiesen, und aus ibm folgt die Richtigkeit des 1. Satzes. In der Tat, wäre der Satz für eine Funktion der Menge $E$ falsch, es müßjte eine Ordnungzahl $\beta$ von der Eigenschaft existieren, daß für alle Funktionen der $\gamma<\beta$ Klassen der Satz richtig wäre, während er für die Funktionen der $\beta^{\text {ten }}$ Klasse unrichtig wäre. $\mathrm{Da}$ aber die Funktionen der $\beta^{\text {ten }}$ Klasse dureh Grenzprozesse aus den. Funktionen der $\gamma\left\langle\beta^{\text {ten }}\right.$ Klassen entstehen, so mulo der Satz 1 auch für die $\beta^{\text {ten }}$ Klasse richtig sein.

2. Satz: Istirgend eine abzählbare Menge von Funktionen der Menge $E$ gegeben, welche auf der Strecke $0-1$ definiert sind, so gibtes dann zu jeder positiven Zahl $\varepsilon<1$ eine perfekte Menge $P$ vom Inhalt 1- - wo alle Funktionendieser Menge stetig sind. ${ }^{1}$ )

In der Tat, da die Menge der Funktionen abzählbar ist, so kann man sie nach dem Typus $\omega$ ordnen. Es seien es die Funktionen $\varphi_{1}(x), \varphi_{2}(x) \ldots \varphi_{n}(x) \ldots$ Es sei außerdem eine Folge von positiven Zahlen $\varepsilon_{1}, \varepsilon_{2} \ldots \varepsilon_{n} \ldots$ gegeben, wo

ist.

$$
\sum_{n=1}^{\infty} \varepsilon_{n}=\varepsilon
$$

Nach dem 1. Satz gibt es eine perfekte Menge $P_{1}$ vom Inhalt $1-\varepsilon_{1}$, wo die Funktion $\varphi_{1}(x)$ stetig ist. Innerhalb der Menge $P_{1}$ gibt es wieder eine zweite perfekte Menge $P_{2}$ rom

1) Allgemein gilt für eine abzäblbare Menge von Funktionen der Menge $E$ der folgende Satz: Ist irgend eine abzählbare Menge $A$ ron Funktionen der Menge $E$ gegeben, welehe auf der Strecke $\overline{0-1}$ definiert sind, so gibtes in jeder perfekten Menge $P$ eine (nirgendwodichte in bezug auf die Menge $P$ ) Menge $P_{1}$, welcho perfekt ist und a af welcher alle Euntionen der Menge A stetig sind.

In der Tat seien $\varphi_{1}(x), \varphi_{2}(x), \ldots \varphi_{n}(x) \ldots$ sämtliche Funktionen 'der Menge $A$ und es sei $x=\chi_{(z)}$ eine eineindeutige stetige Funktion, welche die Strecke $\overline{0-1}$ in die Strecke $\overline{0-1}$ überführt und dabei eine perfekte nirgendwodichte Menge $Q$ vom Inhalt $x>0$ in die Menge $P$ transformiert. "Die Funktionen $x_{1}(z)=\varphi_{1}\{\chi(z)\}, x_{2}(z)=\varphi_{2}(\chi(z)), \ldots x_{n}(z)=\varphi_{n}\{\chi(z)\} \ldots$ sind dann wieder im Sinne $B$ meßbar, es existiert also nach dem 2. Satze auf der Menge $Q$ vom Inhalt $x>0$, eine perfekte nirgendwodichte Menge $Q_{1}$ vom Inhalt $x_{1}<x$, wo alle Funktionen $x_{1}(z), x_{2}(z), \ldots x_{n}(z) \ldots$ stetig sind. Der Menge $Q_{2}$ entspricht bei der. Transformation $z=\varphi(z)$ eine perfekte nirgendwodichte Menge $P_{1}$ (in $x$ ). Man sieht ohne weiteres, $\mathrm{dab}$ auf dieser perfekten Menge $P_{1}$ alle Funktionen $\varphi_{1}(x)$, $\varphi_{2}(x) \ldots \varphi_{n}(x) \ldots$ stetig sein müssen. 
Inhalt $1-\varepsilon_{1}-\varepsilon_{2}$, wo die Funktion $\varphi_{2}(x)$ stetig ist, und allgemein innerhalb der Menge $P_{n-1}$ gibt es eine perfekte Menge $P_{n}$ vom Inhalt $1-\varepsilon_{1}-\varepsilon_{2}-\ldots-\varepsilon_{n-1}-\varepsilon_{n}$, wo die Funktion $\varphi_{n}(x)$ stetig ist. Bezeichnen wir jetzt den Durchschnitt aller dieser perfekten Mengen $P_{1}, P_{2}, \ldots P_{n} \ldots$ mit $Q$, so ist die Menge $Q$ erstens abgeschlossen und zweitens vom Inhalt $\lim _{n=\infty}\left(1-\sum_{m=1}^{m=n} \varepsilon_{m}\right)=1-\varepsilon$. Die abgeschlossene Menge $Q$ ist eine Summe einer abgeschlossenen Menge $A$ und einer perfekten Menge $P$. Es ist also die Menge $P$ vom Inhalt $1-\varepsilon$. Man sieht aber ohne weiteres, daß auf der Menge $P$ jede Funktion der Funktionenmenge $\varphi_{1}(x), \varphi_{2}(x) \ldots \varphi_{n}(x) \ldots$ stetig ist.

Wir wollen jetzt den angekündeten Satz beweisen :

3. Satz: Für die Funktionen zweiter Klasse gibt es keine abzählbare Menge von meßbaren im Sinne $B$ Funktionen, welchedie Eigenschaft hätte, dab jede Funktion der zweiten Klasse durch irgend eine Teilmenge dieser Menge approximierbar wäre. ${ }^{1}$ )

In der Tat existierte eine abzählbare Menge von Funktionen $f_{1}(x), f_{2}(x) \ldots f_{n}(x) \ldots$, welche im Sinne $B$ meßbar wären und welche die verlangte Eigenschaft hätten, so könnte man zu einem Widerspruch kommen. Denn nach den 2 . Satz müßte eine perfekte Menge $P$ vom Inhalt $1-\varepsilon$ existieren, wo alle Funktionen $f_{1}(x)$, $f_{2}(x), \ldots f_{n}(x) \ldots$ stetig wären. Da andrerseits jede Funktion der zweiten Klasse durch irgend eine Teilfolge der Menge $f_{1}(x)$, $f_{2}(x), \ldots f_{n}(x) \ldots$ approximierbar wäre, so mübte jede Funktion der zweiten Klasse auf der perfekten Menge $P$ höchstens punktweise unstetig sein. ${ }^{2}$ ) Das ist aber unmöglich, da es Funktionen der zweiten Klasse gibt, welche auf der Menge $P$ total unstetig sind. Wir zeigen das, indem wir ganz einfach eine solche Funktion konstruieren.

Es sei $A$ eine abzählbare Menge, welche in der Menge $P$ enthalten ist und die Eigenschaft besitzt, daß ihre Ableitung mit der Menge $P$ zusammenfällt. Wir konstruieren dann eine Funktion $f(x)$ folgendermaßen:

$$
\begin{aligned}
& f(x)=1 \text { für die Punkte der Menge } A \\
& f(x)=0 \text { für alle anderen Punkte der Strecke }
\end{aligned}
$$

0-1. Man sieht ohne weiteres, daß die Funktion $f(x)$ von der zweiten Klasse ist und auf der Menge $P$ total unstetig ist. Dadurch ist also der 3. Satz bewiesen.

1) Für Funktionen, welche im Sinne $L$ melibar sind, gilt dasselbe. Es gibt keine abzählbare Menge von im Sinne $L$ meßbaren Funktionen, welche die Eigenschaft hätte, daß jede Funktion der zweiten Klassedurehirgendeine Teilmengedieser Mengeapproximierbar wäre. Dies folgt un mittelbaraus dem Beweis des 3. Satzes, wenn man bemerkt, daB jede im sinne $L$ mebbare Funktion oiner Fonktion zweiter Klasse wesentlich gleich ist.

2) R. Baire: Leçons sur les fonctions discontinues: Seite 80-83. 
4. Satz: Für die Funktionen zweiter Klasse gibt es keine abzählbare Menge von Funktionen, welche die Eigenschaft häte, daß jede Funktion der zweiten Klasse durch eine Teilmenge dieser Menge gleichmäBig approximierbar wäre.

In der Tat bezeichnen wir die abzählbare Menge von Funktionen, $f_{1}(x), f_{2}(x), \ldots f_{n}(x), \ldots$, welche die verlangte Eigenschaft besitzt, mit $A$. Wir wählen dann eine Folge von positiven Zablen $\varepsilon_{1}, \geqq \varepsilon_{2} \geqq \varepsilon_{3} \geqq \cdots \geqq \varepsilon_{n} \geqq \ldots$, wobei $\lim _{n=\infty} \varepsilon_{n}=0$ ist, und bilden eine Folge von Funktionenmengen, die wir mit $A^{(1)}, A^{(2)} \ldots A^{(n)} \ldots$ bezeichnen. Jede Funktionenmenge $A^{(n)}$ hat folgende Eigensehaften; erstens ist sie eine Teilmenge der Menge $A$, zweitens ist $f_{x}^{(n)}(x)$ irgendeine Funktion der Menge $A^{(n)}$, so existiert mindestens eine Funktion $\varphi_{x}^{(n)}(x)$ von der zweiten Funktionenklasse derart, daß

$$
\left|f_{*}^{(n)}(x)-\varphi_{*}^{(n)}(x)\right|<\varepsilon_{n}
$$

ist (für alle Punkte der Strecke $\overline{0-1}$ ).

Bezeichnen wir die Funktion $f_{x}^{(n)}(x)-\varphi_{z}^{(n)}(x)$ mit $\varepsilon_{x}^{(n)}(x)$, so ist $f_{x}^{(n)}(x)=\varphi_{x}^{(n)}(x)+\varepsilon_{x}^{(n)}(x)$, wobei $\left|\varepsilon_{x}^{(n)}(x)\right| \leqq \varepsilon_{n}$ ist.

Es sei $A^{(\omega)}$ die Menge aller Funktionen, welche in mindestens einer der Funktionenmenge $A^{(1)}, A^{(2)}, \ldots A^{(n)} \ldots$ vorkommen. Die Menge $A^{(\omega)}$ ist dann auch eine Teilmenge der Menge $A$, und hat dieselbe Eigensehaft, wie die Menge $A$, d. i. jede Funktion $\varphi(x)$ der zweiten Funktionenfolge wird durch irgendeine Teilmenge der Menge $A^{(\omega)}$ gleichmäßig approximiert. In der Tat ist $\varphi(x)$ irgendeine Funktion der zweiten Funktionenklasse und ist $f_{n_{1}}(x), f_{n_{2}}(x) \ldots$ $f_{n_{z}}(x), \ldots$ eine Teilmenge der Menge $A$, welche die Funktion $\varphi(x)$ gleichmälifig approximiert, dann gibt es $z u$ der Zahl $\varepsilon_{1}$ ein Index $n_{r}$ von der Eigenschaft, daßs

$$
\left|\varphi(x)-f_{n_{m}}(x)\right| \leqq \varepsilon_{1}
$$

ist, wenn $n_{m} \geqq n_{r}$ ist (für alle Punkte der Streche $\overline{0-1}$ ). Es gehören dann also alle Funktionen $f_{n_{r}}(x), f_{n_{r+1}}(x) \ldots f_{n_{m}}(x), \ldots$ wobei $n_{m}>n_{2}$ ist, der Menge $A^{(\omega)}$ und approximieron gleichmähig die Funktion $\varphi(x)$.

Andrerseits sieht man ohne weiteres, daß die Funktionenmenge $\varphi_{*}^{(n)}(x)$, wo $n==1,2, \ldots m \ldots$ und $x=1,2, \ldots m \ldots$ ist, auch dieselbe Eigenschaft besitzt, wie die Funktionenmenge $A^{(\omega)}$. In der Tat, es ist $f_{n_{m}}(x)=\varphi_{n_{m}}(x)+\varepsilon_{n_{m}}(x)$, wo die Funktionen $\varphi_{n_{m}}(x)$ 
und $\varepsilon_{n_{n}}(x)$ Funktionen der Menge $\varphi_{x}^{(n)}(x)$ und $\varepsilon_{x}^{(n)}(x)$ sind ${ }^{1}$ ), wo $n=1$, $2 \ldots m \ldots$ und $x=1,2, \ldots m \ldots$ ist.

Andrerseits ist irgendeine positive Größe $\varepsilon$ gegeben, welche kleiner als die Größe $\varepsilon_{l}$ ist, so können wir immer einen Index $n_{r}$ finden von der Eigenschaft,

$$
\left|\varphi(x)-f_{n_{m}}(x)\right| \leqq \varepsilon_{l}
$$

ist (für alle Punkte der Strecke $\overline{0-1}$ ), wenn nur $n_{m} \geq n_{x}$ ist. Es ist aber $f_{n_{m}}(x)=\varphi_{n_{m}}(x),+\varepsilon_{n_{m}}(x)$, also

$$
\left|\varphi(x)-\varphi_{n_{m}}(x)\right| \leqq\left|\varepsilon_{n_{m}}(x)\right|+\varepsilon_{l} .
$$

Da aber $\left|\varepsilon_{n_{m}}(x)\right|<\varepsilon_{l}$ sein $\mathrm{mul}$, so ist

$$
\left|\varphi(x)-\varphi_{n_{m}}(x)\right|<2 \varepsilon_{l}
$$

(für alle Punkte der Strecke $\overline{0-1}$ ).

Fs hat also die Menge der Funktionen $\varphi_{x}^{(n)}(x)$, wo $n=1$, $2, \ldots m \ldots$ und $x=1,2, \ldots m \ldots$ ist, dieselbe Eigenschaft, wie die Funktionenmenge $A^{(\omega)}$, und da diese Menge auch abzählbar ist und alle Funktionen $\varphi_{x}^{(n)}(x)$ der zweiten Funktionenklasse angehören, so ist das nach dem 3. Satz unmöglich. Dadurch ist also der 4. Satz bewiesen.

Wir wollen jetzt einen Satz über die Funktionen erster Klasse beweisen:

5. Satz: Es existiert eine abzählbare Menge von Funktionen $f_{1}(x), f_{2}(x), \ldots f_{n}(x) \ldots$, welche folgende Eigensehaft besitzt: ist $\varphi(x)$ irgend eine Funktion der ersten Klasse, so kann man eine Teilmengevon Funktionen $f_{n_{1}}(x), f_{n_{2}}(x), \ldots f_{n_{x}}(x) \ldots$ aus der Menge $f_{1}(x), f_{2}(x), \ldots f_{n}(x), \ldots$ herausgreifen, so dab diese Teilfolge gegen die Funktion $\varphi(x)$ konvergiert.

In der Tat sei $f_{1}(x), f_{2}(x), \ldots f_{n}(x) \ldots$ eine abzählbare Menge von stetigen Funktionen, welche die Eigenschaft besitzt, daß jede stetige Funktion gleichmäßig durch irgend eine Teilmenge dieser Menge approximiert wird. Es ist dann sehr leicht zu beweisen, daß diese Menge auch alle Funktionen der ersten Klasse approximiert. Ist nämlich $\varphi(x)$ irgend eine Funktion der ersten Klasse, so existiert

${ }^{1)}$ und zwar sollen die Funktionen $\varphi_{n_{m}}(x)$ und $\varepsilon_{n_{m}}(x)$ folgendermaßen gewählt werden: ist $\left|\varphi(x)-f_{n_{m}}(x)\right| \leqq \varepsilon_{r}$ (für alle Punkte der Strecke $\overline{0-1}$ ) aber nicht $\left|\varphi(x)-f_{n_{m}}(x)\right| \leqq \varepsilon_{r+1}$ (für alle Pankte der Strecke $\overline{0-1}$ ), so sollen die Funktionen $\varphi_{n_{m}}(x), \varepsilon_{n_{m}}(x)$ mit irgendeiner Funktion $\varphi_{1}^{(r)}(x), \varphi_{2}^{(r)}(x) \ldots$ $\varphi_{n}^{(r)}(x) \ldots, \varepsilon_{1}^{(r)}(x), \varepsilon_{2}^{(r)}(x), \ldots \varepsilon_{n}^{(r)}(x) \ldots$, , (und auch die Funktion $f_{n_{m}}(x)$ mit irgendeiner Funktion der Menge $A^{(r)}$ ) zusammenfallen. 
laut der Definition dieser Funktion eine Folge von stetigen Funktionen $\varphi_{1}(x), \varphi_{2}(x), \ldots \varphi_{n}(x) \ldots$, welche gegen die Funktion $\varphi(x)$ konvergiert. Es ist also

$$
\lim _{n=\infty} \varphi_{n}(x)=\varphi(x) \text {. }
$$

Es sei $\varepsilon_{1} \geqq \varepsilon_{2} \geqq \ldots \geqq \varepsilon_{n} \geqq \ldots$ eine Folge positiver Zahlen, wobei $\sum_{n=1}^{\infty} \varepsilon_{n}=\varepsilon$ ist, dann kann man zu jeder stetigen Funktion $\varphi_{\varkappa}(x)$ und jeder positiven Zahl $\varepsilon_{\varkappa}$ eine stetige Funktion $f_{n_{x}}(x)$ $f_{n_{\varkappa}}(x)$ aus der Menge $f_{1}(x), f_{2}(x), \ldots, f_{n}(x), \ldots$ herausgreifen, so dab

$$
\left|f_{n_{x}}(x)-\varphi_{x}(x)\right|<\varepsilon_{x}
$$

ist. Wir wollen zoigen, dab

$$
\lim _{x=\infty} f_{n_{x}}(x)=\varphi(x)
$$

ist. In der Tat, ist $x_{0}$ irgend ein Punkt, so existiert zu jeder positiven Zahl $\varepsilon$ eine naturliche Zahl $r$ von der Eigenschaft, daß

$$
\left|\varphi_{m}\left(x_{0}\right)-\varphi\left(x_{0}\right)\right|<\varepsilon
$$

ist, wenn $m \geqq r$ ist. Anderseits ist aber

es ist also

$$
\left|f_{n_{m}}\left(x_{0}\right)-\varphi_{m}\left(x_{0}\right)\right|<\varepsilon_{m}
$$

$$
\left|f_{n_{m}}\left(x_{0}\right)-\varphi\left(x_{0}\right)\right|<\varepsilon+\varepsilon_{m} \leqq \varepsilon+\varepsilon_{r}<2 \varepsilon
$$

für alle $n_{m} \geq n_{r}$. Dadurch ist der 5 . Satz bewiesen.

Aus dem 2. Satz folgt noch weiter ein Satz über die Funktionen der ersten Klasse.

6. Satz: Es existiert keine abzählbare Menge von Funktionen, $f_{1}(x), f_{2}(x), \ldots f_{n}(x), \ldots$, welche folgende Eigenschaft besitzt; ist $\varphi(x)$ irgend eine Funktionder ersten Klasse, sokannmannichta der Menge der Funktionen $f_{1}(x), f_{2}(x), \ldots f_{n}(x) \ldots$ eine Teilmenge $f_{n_{1}}(x), f_{n_{x}}(x) \ldots f_{n_{n}}(x) \ldots$ herausgreifen, so dab diese Teilfolge gegen die Funktion gleichmäßig konvergiert.

In der Tat, wäre das der Fall und wende man die Beweisführung des Satzes 4 an, so müßte eine perfekte Menge $P$ vom Inhalt $x>0$ existieren, wo alle Funktionen $\varphi_{x}^{(x)}(x)$ der ersten Klasse stetig wären. Dies ist aber unmöglich, da sonst alle Funktionen der ersten Klasse auf der Menge $P$ stetig wären. $\mathrm{E}_{\mathrm{s}}$ ist also der 6. Satz richtig. ${ }^{1}$ )

1) Es existieren nämlich Funktionen excter Klasse, wolche auf der perfelkten Menge $P$ panktweise unstetig sind. 


\section{Anhang.}

Wir wollen in den. Anhange ein Beispiel einer kompakten, abgeschlossenen Funktionenmenge $T$ geben, für welche das Abzählbarkeitsprinzip ${ }^{1}$ ) nicht gilt. Wir zeigen dies unter der Voraussetzung der Wohlordnung des Kontinuums. In der Tat läßt sich das Kontinuum unter der Voraussetzung der Wohlordnung in $\mathbf{\aleph}_{\nu}=c$ nichtmeßbare Mengen spalten. ${ }^{2}$ ) Wir ordnen diese Menge der nichtmeßbaren Mengen $M_{1}, M_{2} \ldots$ nach dem Typus $\Omega_{\nu}$. Es ist also:

$$
S=\left\{M_{1}, M_{2}, \ldots M_{n} \ldots M_{\omega} \ldots M_{\varepsilon} \ldots M_{\varsigma} \ldots\right\}
$$

wo $\varepsilon, 5 \ldots<\Omega_{y}$ ist.

Ist $\varepsilon$ irgend eine Ordnungszahl, welche kleiner als $\Omega_{v}$ ist, so ordnen wir ihr die Menge $P_{\varepsilon} \mathrm{zu}$, wo

$$
P_{\varepsilon}=M_{1}+M_{2}+\ldots+M_{\omega}+\ldots+M_{\varsigma}+\ldots
$$

ist, also wo die Menge $P_{\varepsilon}$ alle Mengen $M_{s}$ enthält, deren Index $\varsigma$ kleiner als die Ordnungszahl $\varepsilon$ ist. Wir ordnen jetzt jeder Ordnungszahl $\varepsilon$ eine Funktion $f_{\varepsilon}(x)$ folgendermaßen:

$$
\begin{aligned}
& f_{\varepsilon}(x)=1 \text { für all } 3 \text { Punkte der Menge } P_{\varepsilon} \\
& f_{\varepsilon}(x)=0 \text { für alle anderen Punkte der Strecke }
\end{aligned}
$$

$\overline{0-1}$. Man sieht obne weiteres, daß jede der Funktionen $f_{\varepsilon}(x)$ nichtmeßbar im Sinne $L$ ist, da jede der Mengen $P_{\varepsilon}$ nichtmeßbar ist. Anderseits sieht man ohne weiteres auch, daß die Menge der Funktionen $f_{2}(x), f_{2}(x) \ldots f_{n}(x) \ldots f_{\omega}(x), \ldots f_{\varepsilon}(x), \ldots$, die wir mit $T$ bezeichnen, kompakt und abgeschlossen ist.

In der Tat, ist irgendeine unendliche abzählbare Menge $T^{\prime \prime}$ von Funktionen, die in der Menge $T$ enthalten ist, so ordnen wir sie zuerst nach dem wachsenden Index, es sei also

$$
T^{\prime}=\left\{f_{\alpha}(x), f_{\beta}(x), \ldots f_{\nu}(x) \ldots f_{\lambda}(x) \ldots\right\}
$$

wo $\alpha \prec \beta<\ldots<\gamma \prec \ldots<\lambda \prec \ldots<\Omega_{v}$ ist. Aus der Menge der Indices $\alpha, \beta, \ldots \gamma \ldots \lambda \ldots$ kann man eine Teilmenge von Indices heraus greifen, welche den Ordnungstypus $\omega$ besitzt. Es sei das die Folge $\alpha^{\prime}, \alpha^{\prime \prime} \ldots \alpha^{\prime \prime \prime} \ldots \alpha^{(n)} \ldots$; die Menge der Funktionen $f_{\alpha^{\prime}}(x), f_{\alpha^{\prime \prime}}(x) \ldots$ $f_{\alpha}^{(n)}(x)$. . besitzt dann eine Grenze, und zwar ist das die Funktion $f_{\xi}(x)$ wo $\xi=\lim \alpha^{(n)}$ ist, wie man $\xi$ sehr leicht einsehen kann. Es ist also die Menge $\stackrel{n}{T}$ kompakt und abgeschlossen.

1) d. i. es existiert eine abzählbare Menge von Funktionen, welche die Eigenschaft besitzt, daß ihre Teilmengen jede Funktion der vorgegebenen Menge $T$ approximieren.

2) C. Burstin: Die Spaltung des Kontinuums in $c$ im Sinne $L$ nichtmeßbare Mengen. Sitzber. der Kaiserl. Akad. in Wien, Bd. 125, 3. Heft, Abt. II a. 
Wir wollen jetzt noch zeigen, daß für diese Funktionenmenge das Abzählbarkeitsprinzip nicht gilt. Die Behauptung folgt unmittelbar aus dem 2. Satz, indem man eine dem 4. Satze analoge Beweisführung auf die Funktionenmenge $T$ anwendet. In der Tat sei $\varphi_{1}(x), \varphi_{2}(x) \ldots \varphi_{n}(x) \ldots$ diese abzählbare Menge von Funktionen, dann wählen wir eine positive Größe $\varepsilon<\frac{1}{4}$ und greifen aus dieser abzählbaren Menge nur solche Funktionen heraus, für welche mindestens eine Funktion der Menge $T$ existiert, die von irgendeiner Funktion $\varphi_{1}(x) \ldots \varphi_{n}(x) \ldots$ sich nur um Größe $\varepsilon$ auf irgendeiner Menge vom Inhalt $\frac{1}{2}+\delta(\delta>0)$ unterscheidet. Die so gebildete Funktionenmenge hat dieselbe Eigensehaft wie die Menge der Funktionen $\varphi_{1}(x), \varphi_{2}(x), \ldots \varphi_{n}(x) \ldots$ Bezeichnen wir diese Funktionen mit $\varphi_{1}^{(1)}(x), \varphi_{2}^{(1)}(x), \ldots \varphi_{n}^{(1)}(x) \ldots$, dann muß mindestens eine Funktion $\varphi_{p}^{(1)}(x)$ existieren von der Eigenschaft, daß sie mindestens zwei Funktionen $f_{a}(x), f_{\beta}(x)$ der Menge $T$ approximiert, und zwar so, daßo

$$
(I)\left|\varphi_{x}^{(1)}(x)-f_{a}(x)\right|<\varepsilon \text { und }(I I)\left|\varphi_{x}^{(1)}(x)-f_{\beta}(x)\right|<\varepsilon
$$

ist, auf zwei Mengen vom Inhalt $\frac{1}{2}+\delta$. Diese zwei Mengen haben eine Menge $P$ gemeinsam, welche mindestens vom Inhalt $\delta$ ist, und für diese Mengen gelten beide Ungleichungen $(I)$ und $(I I)^{\circ}$ zugleich. Aus $(I)$ und $(I I)$ folgt dann für die Menge $P:\left|f_{\alpha}(x)-f_{\beta}(x)\right|<2 \varepsilon<\frac{1}{2}$. Dies ist aber unmöglich, da auf der Menge $D\left(P_{\beta}-P_{\alpha}, P\right)$, $\left|f_{\alpha}(x)-f_{\beta}(x)\right|=1$ ist (wobei $\beta>\alpha$ ist), wie man ohne weiteres aus der Konstruktion der Funktionen $f_{\varepsilon}(x)$ einsehen kann. Es ist also unsere Behauptung richtig. $\left.{ }^{1}\right)$

1) Ist $c>\$_{1}$, so können wir innerhalb der Funktionenmenge $T$ eine Teilmenge von der Mächtigkeit $\aleph_{1}$ herausgreifen, und zwar es sei das der erste $A b-$ schnitt innerhalb der Funktionenmenge $T$, der die Mächtigknit $\aleph_{1}$ besitzt. Wir bezeichnen diesen Abschnitt mit $A$. Man sieht ohne weiteres, dab die Funktionenmenge kompakt und abgeschlossen ist. Wir haben also 1. unter der Voraussetzung der Wohlordnung des Kontinuums und 2. unter der Voraussetzung, daß $c>\aleph_{1}$ ist, eine abgeschlossene Funktionenmenge $A$ definiert, welche nicht abzählbar und von der Mächtigkeit $\aleph_{1}<c$ ist und für diese Menge gilt das Abzählbarkeitsprinzip auch nicht, wie man ohne weiteres einsehen kann. 